\title{
An Innovative Structural Solution to Failed Stabilized Earth Embankment in Multilevel Interchange
}

\author{
Ali Kara \\ akara@ashghal.gov.qa \\ Highway Projects Department, Public Works Authority (Ashghal), Doha, Qatar \\ Tamer Tahoun \\ tamer.tahoun@parsons.com \\ Parsons International, Doha, Qatar
}

\begin{abstract}
This paper summarizes a case study of a failure in a mechanically stabilized earth retaining wall ramp heading to a multilevel interchange. The failure was caused by a washout cavity resulting from a long-term leakage from unknown existing wet utilities adjacent to the mechanically stabilized earth retaining wall. The interchange is located within a very congested traffic area and has limited right of way and working space. The methodology of investigation, root cause of the failure, evaluation of the existing structure and innovative solution to the problem within fast-track design and construction are studied in this paper. Due to the necessity of maintaining the existing traffic movements at the interchange which is located at one of the most important expressway routes, timely rectification methodology was conceived, designed and implemented. This paper is prepared only to evaluate the technical innovative alternatives, solutions and fast-track construction without mentioning the interchange and project area.
\end{abstract}

Keywords: Embankment failure in MSE wall; Fast track rectification; Wet utility damages

\section{INTRODUCTION}

The major objective of this paper to outline the rectification method of the partial failure on the Mechanically Stabilized Earth Wall ( MSE ) Embankment ramp heading the bridge in addition to the evaluation of all bridge ramps, pavement, service roads considering a major constraint which is the time limitation to execute the work since the project under study is located in one of the critical traffic flows. The country and the region of the project area are not mentioned in this paper.

The multilevel interchange is located within a traffic congested area and having multi direction movements providing expressway functionality and local access. One of the MSE ramp heading bridge had pavement failure, which was observed by local commuters. The relevant traffic department stopped the traffic and detour the traffic movement to local roads. Relevant authority professional team visited the site and initiated a fast track investigation of the failure along with ultimate design and construction solution proposals.

The failure was in the pavement along a deep crack in the backfilling of the ramp embankment as shown in figure 1 . 

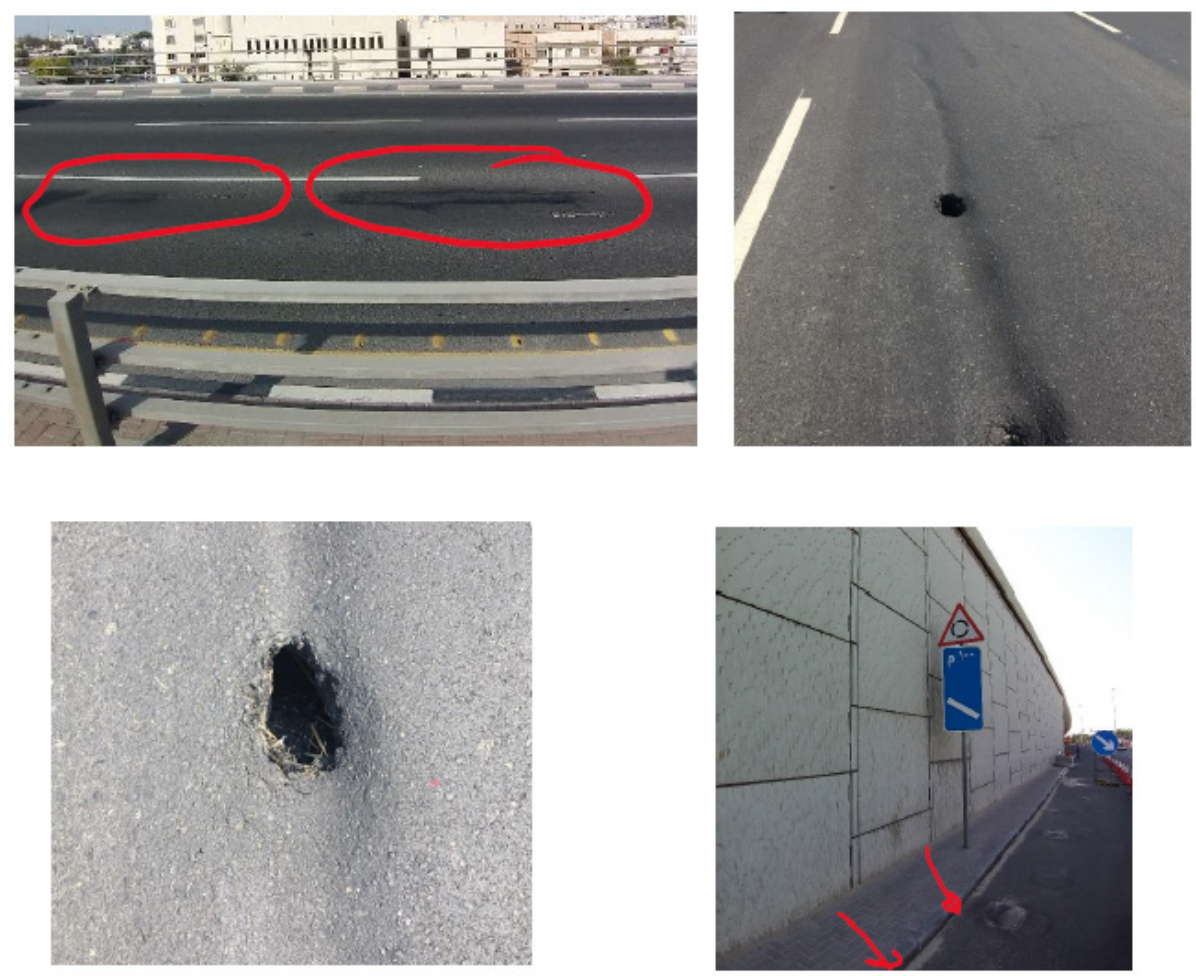

Figure 1: Partial Failure

The site investigation started with a field geophysical survey. However, due to the necessity of maintaining the existing traffic, the geotechnical and geophysical report was delivered progressively in parallel to the construction.

Following field investigations were carried out:

- Geotechnical and Geophysical survey using MASW covering all the embankments,

- Intrusive works at locations identified from the MASW investigation and complemented with a GPR survey,

- Pavement evaluation using destructive and non-destructive evaluation.

Destructive testing included pavement cores and trial to evaluate the material the layer thickness properties. The non-destructive testing using Falling Weight Deflectometer (FWD) was carried out on the bridge ramps and the service road. The purpose of FWD was to evaluate the existing structural capacity of the pavement.

\section{PROJECT DESCRIPTION}

The Project investigation area was limited within the interchange embankments approach ramps, Mechanically Stabilized Earth Walls (MSE), and service road at ground along MSE walls.

The MSE wall of embankment approach ramp was deflected by $50 \mathrm{~mm}$ horizontally measured from top within 50m length. Deep cracks were observed in the asphalt and embankment fill. A vertical settlement of $30 \mathrm{~mm}$ was observed at the MSE wall. The directly affected area within the ramp is an open crack of around $16 \mathrm{~m}$ length and $2 \mathrm{~m}$ 
depth. This crack is suspected to extend around $14 \mathrm{~m}$. Geotechnical investigation and geophysical survey were undertaken to obtain detailed information about the surface and subsurface strata allowing the condition assessment of the MSE wall embankment.

\section{GEOTECHNICAL INVESTIGATION AND DESIGN}

\subsection{Geotechnical Investigation and Geophysical Survey}

The geotechnical investigation and geophysical survey undertaken to MSE wall embankment approach include:

- Walk-over survey and gathering of chronological data of the affected zone,

- Geophysical survey (MASW) to determine the weak areas and zones of risk within the MSE wall and adjacent road formation.

- Based on results of walk-over and geophysical survey, the below geotechnical investigation was undertaken:

i. Drilling of 4 diagraph boreholes down to $15 \mathrm{~m}$ below ground level (BGL) in the close vicinity of MSE wall along services road.

ii. Drilling two geotechnical boreholes down to $15 \mathrm{~m} \mathrm{BGL}$ on the embankment in the close vicinity of MSE wall. Carrying out SPT tests in boreholes.

iii. 3 DCPT within crack area.

\subsection{Findings from Geotechnical Investigation and Geophysical Survey}

Based on the investigations carried out for the MSE wall of embankment, the following conclusions are drawn:

1. The identified velocities within the embankment backfill corresponds to generally medium dense to dense material with shear wave velocities between $180 \mathrm{~m} / \mathrm{s}$ and $360 \mathrm{~m} / \mathrm{s}$ (SPT results also show the similar conditions) while the rock is identified at around $+7.0 \mathrm{~m}$ to $+8.0 \mathrm{~m}$ where the shear wave velocities exceed $600 \mathrm{~m} / \mathrm{s}$ which is line with the conditions revealed from the geotechnical boreholes.

2. The rock contains some weak areas, which are attributed to existing utilities. The backfill exhibits some lower SPT values close to the service road elevation (within limited thickness of $0.5 \mathrm{~m}$ ). The service road top $1.5 \mathrm{~m}$ to $2.0 \mathrm{~m}$ near the MSE wall contains loose sandy material susceptible to wash out if in contact with moving water (rain or broken wet utilities). This effectively resulted in the partial failure of the MSE wall of the south-west embankment. The MSE wall is intact with no apparent face distortion or horizontal movement within the strips zone.

3. The repetitive migration of dune sand under the adjacent service road due to several leaks and movement of water, resulting in partial loosening of the foundation ground under the outer part of the MSE wall is the likely cause of bearing capacity failure of the MSE wall in addition to the washout cavities as shown in figure 2. 


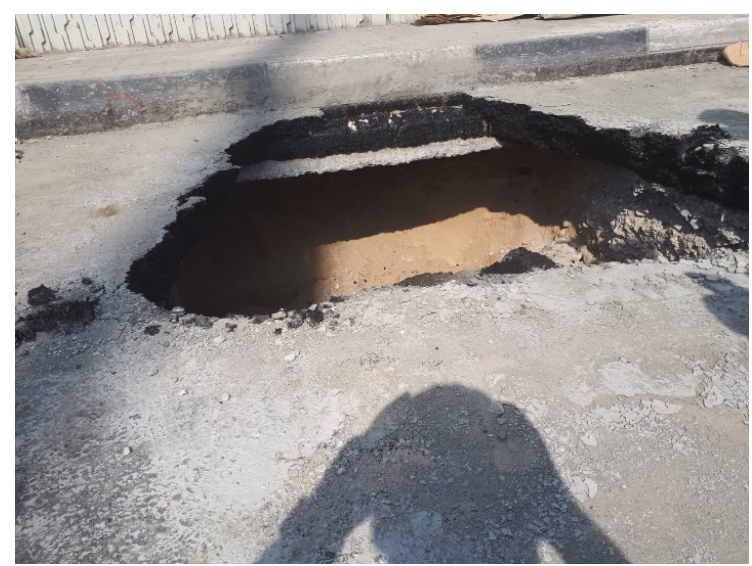

Figure 2: Washout Cavities

Accordingly, it was concluded that the partially failed section in the MSE wall at the side is typical to tilt/bearing capacity failure mode as described by (Bobet, 2002), see Figure 3

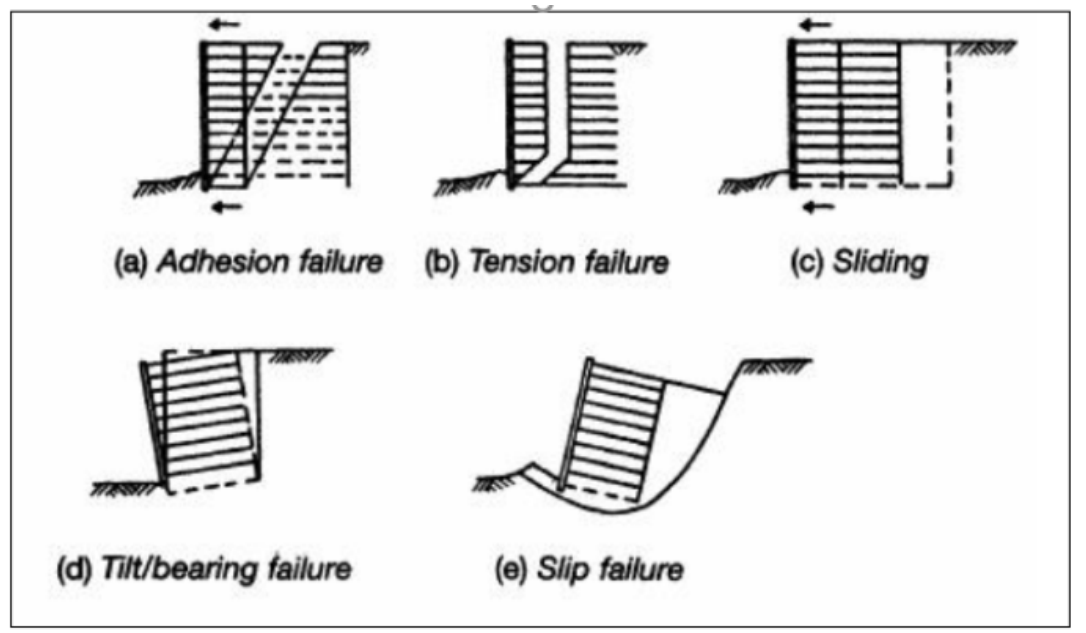

Figure 3: MSE Walls Typical Failure, adapted from (Bobet, 2002)

The likely cause of the bearing capacity failure is the repetitive migration of dune sand under the adjacent road due to several leaks and movement of water, resulting in partial loosening of the foundation ground under the outer part of the MSE wall. The movement took place at the current location since the foundation fill, because of loosening, could not carry anymore the load/height of the MSE wall. On the other hand, the sections towards the lower level of the embankment, which are mostly affected by the migration of the sand with several wash out cavities, did not fail due to their reduced height (less than $2 \mathrm{~m}$ ).

Although all the MSE walls (except the partially failed section) are in good condition, there was a risk of washout and material loosening along the service road adjacent to the MSE walls foundation, spotted from the various trial pits, boreholes, MASW survey and the GPR survey. 


\subsection{Geotechnical Design Parameters \& Recommendations}

Based on the above investigation, the recommendations for the subsurface properties and solutions are given below:

1. The rock-head below MSE wall is at level varying from $+8.0 \mathrm{~m}$ to $+9.5 \mathrm{~m}$ whereas it is around $+7.75 \mathrm{~m}$ below service road.

2. Table 1 depicts the design parameters as recommended in the geotechnical report:

Table 1: Design Parameters of Limestone Rock

\begin{tabular}{|l|l|l|l|l|l|}
\hline RQD (\%) & GSI & Density (kN/m3) & PLI (MPa) & UCS (MPa) & E (MPa) \\
\hline 20 & 30 & 20.5 & 0.7 & 7.0 & 2000 \\
\hline *taking into account the point load index values with a ratio of 10 to the UCS \\
\hline
\end{tabular}

3. The pile ultimate skin friction is recommended at $1000 \mathrm{kPa}$.

4. The piles should be tested in accordance international specifications.

5. The pile socket into bedrock should be verified on site through monitoring of the cutting and logging of boring speed.

\subsection{Rectification Structural Design}

The structural design was carried out using BS 5400 and BD 347/01. Multiple options were investigated to provide a rectification of the MSE walls partial failure. Further investigations of the design studies resulted in three viable options.

The first option was to provide grout injection at the MSE wall along with rebuilding the top two meters that experienced a deep pavement crack, please refer to Figure 4.

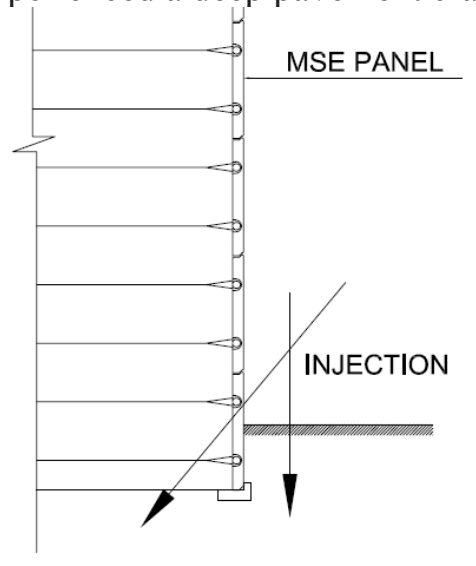

Figure 4: Proposed First Option

This option was not utilized due to the associated uncertainties of the size of the washout cavities that the geophysical surveys indicated. The second option was to rebuild an independent structure consisting of piles and pile cap that carries out the backfilling at the 90 meters length that experienced most of the cavities, Figure 5. 


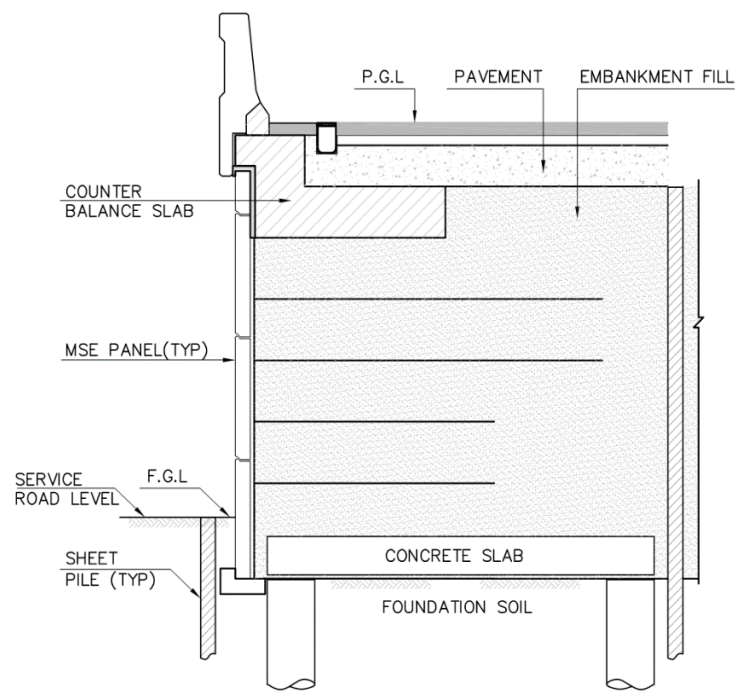

Figure 5: Proposed Second Option

This option was not utilized due to the time limitation which was set as one month for the design and construction. The time constraint for the rectification was the biggest constraint that did not allow to excavate significant depth, build piles and pile cap, then backfill the area under consideration.

Due to the observations of the large cavities at the MSE service road level right below the MSE walls and the partial loosening of the foundation ground under the outer part of the MSE wall, a complete independent structure at this area has been introduced as a third option. The structural system consists of slab supported on two rows of piles. These piles at the outer part have been casted contiguously from the external side to retain the MSE wall fill and relieve any earth pressure transfer to the MSE walls under study while the piles at the other side of MSE wall have been casted at 2.1 meters center to center. This work has been selected to be carried out for the affected area. The advantage of this option is the time saving of deep excavation along with high backfilling, Figure 6, 7 , and 8 .

The construction sequence has been selected and implemented at site considering the necessity of supporting the MSE wall panels during the piles casting due to the expected damage of MSE walls straps resulting from the piling work.

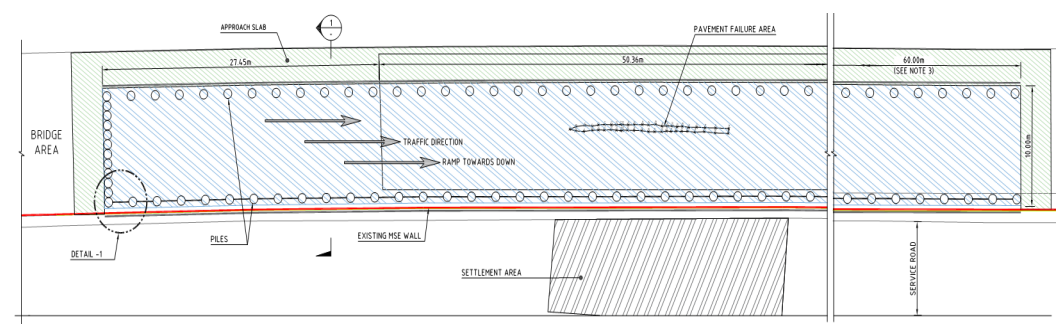

Figure 6: Utilized Option - Layout 


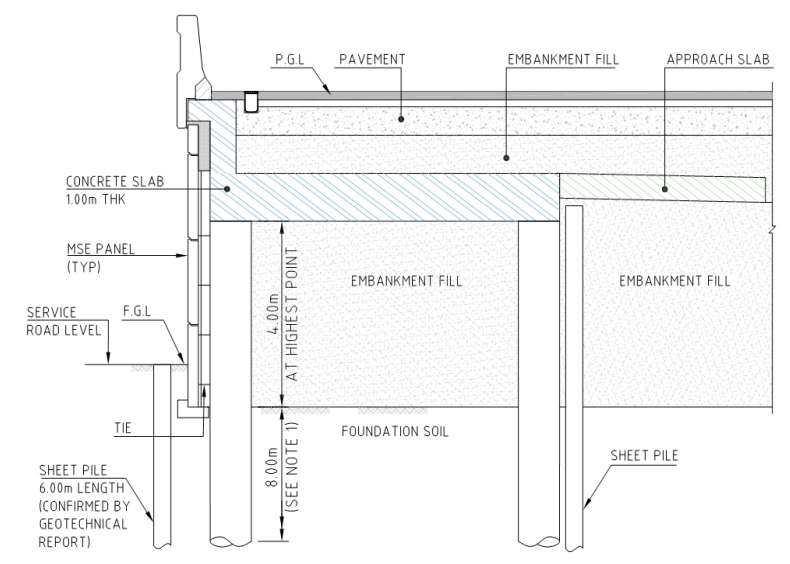

Figure 7: Utilized Option - Section 1-1

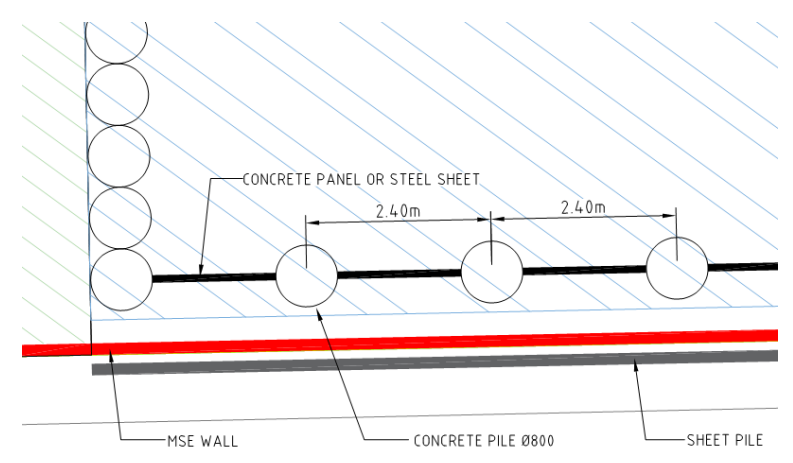

Figure 8: Utilized Option - Detail -1

\section{PAVEMENT}

The Pavement Design is done keeping in view the future anticipated traffic volumes. This included the bridge ramps and service roads. For the upgradation of existing pavement, two options are considered; full reconstruction option and mill/overlay option. The upgradation option for a given pavement section was selected based on the existing road quality (surface distress) and structural capacity of the pavement section. The existing pavement structural capacity was evaluated using both destructive and nondestructive approaches.

Pavement study was carried out based on the condition of the existing pavement and to provide an estimated overlay requirement to extend the structural life of the pavement for 20 years. In addition, FWD testing was also used for the identification of the weak areas.

Coring of the existing pavement for the estimation of asphalt thickness along with Falling Weight Deflectometer (FWD) analysis was carried for the embankments ramp up and ramps down. Towards the direction failed pavement because of the partial movement of MSE wall. For this section full depth pavement, section was proposed. 


\section{CONCLUSION AND RECOMMENDATIONS}

Although the rectifications for the affected areas have been carried out, the below recommendations are being shared to avoid the reoccurrence of the MSE walls partial failure in the future:

- The repair was carried out by the contractor for the service road at the areas where poor backfilling was captured, it was recommended to divert the utilities away from the MSE walls considering the limitation of the boreholes, trial pits, and geo-physical survey to capture every single area that had poor backfilling in the past.

- Any leakage of the wet utilities at the MSE wall or nearby should be treated immediately.

- As another level of mitigation, vertical permanent shoring could take place adjacent to the MSE wall at the service road level to assure that there will be no impact from the service road backfilling on the MSE walls.

- Regular inspection of the MSE wall to observe any deformation that might occur.

- Regular inspection of the pavement to report any deformation at the bridge level or at the service road if took place.

\section{REFERENCES}

Bobet, A. (2002). Design of MSE Walls for Fully Saturated Conditions (Report No. FHWA/IN/ JTRP-2002/13). Perdu University, Indiana, the USA.

British Standards Institution (1990). BS5400-4. Steel, Concrete, and Composite Bridges: Code for Practice for Design of Concrete Bridges, the UK.

Design Manual for Road and Bridges (2001). BD37/01. Load for Highway and Bridges: Highway Structures: Approval Procedures and General Design, the UK. 